\title{
Orbital Pseudotumour
}

\author{
Lt Col V Maurya*, Lt Col S Gautam ${ }^{+}$
}

MJAFI 2007; 63 : 292-293

Key Words : Orbital pseudotumour

$\mathrm{O}_{\mathrm{s}}^{+}$ rbital inflammatory syndrome commonly termed as pseudotumour is characterized by idiopathic inflammation of the orbital tissues, affecting adults and children, unrelated to thyroid related orbitopathy or other systemic disorders. Patients with orbital inflammatory syndrome typically present with orbital pain, restricted movement, diplopia, proptosis and impaired vision with optic nerve involvement [1]. We report here a case of orbital pseudotumour in an adult.

\section{Case report}

A 28 year old serving soldier presented with complaints of pain, diplopia, and proptosis left eye. On examination there was mild proptosis, conjuctival congestion and restricted movements of left eye. The corneas were clear. No erythema of the lids was appreciated. Anterior chambers were clear and vision was normal. Intraocular pressure and fundus examination was normal. Patient was clinically diagnosed as a case of orbital pseudotumour. Routine laboratory investigation including T3, T4, and TSH were normal. Contrast enhanced computed tomography (CECT) orbit in axial and coronal plane showed well defined soft tissue density enhancing retrobulbar mass involving the belly and tendinous insertion of left inferior rectus muscle resulting in proptosis (Figs. 1,2). Patient was treated with oral corticosteroids in tapering doses for three months. He showed rapid clinical improvement with complete remission of signs and symptoms. Follow up commuted tomography (CT) scan showed reduction in the size of inflammatory mass with residual thickness of the left inferior rectus muscle with no proptosis.

\section{Discussion}

Orbital pseudotumour (OP) or idiopathic non-specific orbital inflammation is a non-infectious acute inflammation of the orbits that presents with orbital swelling or a mass. This descriptive term was first used in 1905 by Birch-Hirschfield to describe an inflammatory condition of the orbit of unknown aetiology. It therefore excludes specific infections, granulomatous diseases and collagen-vascular disorders such as polyarteritis nodosa and Wegener's granulomatosis [2]. The clinical picture of pseudotumour varies widely with single or combined signs of mass effect, inflammation and infiltration. OP may present as proptosis, eye pain, ptosis, lid oedema, conjunctivitis with or without chemosis (swelling of the conjunctiva) and limitation of extraocular movements [1]. Unilateral orbital involvement with fever, headaches and eye discharge are common. Orbital CT and magnetic resonance imaging (MRI) are the important diagnostic tests, but serologic studies are necessary to exclude a systemic cause. Biopsy is usually avoided, to minimise the risk of damage to vital structures within the orbit. On CT, the pseudotumour reveals a broad range of pathological changes [3]. It may appear as an isolated discrete lesion with well-defined borders resembling neoplasm or specific granuloma. The inflammatory changes may remain localised to part of the globe resulting in an apparent thickening of the scleral margin. Myositic pseudotumour can mimic Grave's disease but in pseudotumour the involvement is less regular and unilateral with involvement of tendinous insertion of extraocular muscles [4]. Infiltrative changes in the adjacent fat, periocular involvement or a discrete mass with thickened muscles favours the diagnosis of pseudotumour. Varying degrees of contrast enhancement of the abnormal soft tissue is seen in most cases of pseudotumours.

On magnetic resonance imaging (MRI), these lesions are usually hypointense on $\mathrm{T} 2 \mathrm{~W}$ images and show homogeneous contrast enhancement. The lack of mobile protons due to the fibrotic background and high cellularity of the lesions may be the reason for their hypointensity and weaker enhancement on MR images. The histopathology of orbital pseudotumour shows polymorphic lymphocytic and plasmacytic infiltrates and eosinophilia with fibrosis of varying degrees. Contrary to an old belief, orbital pseudotumour is not related to 


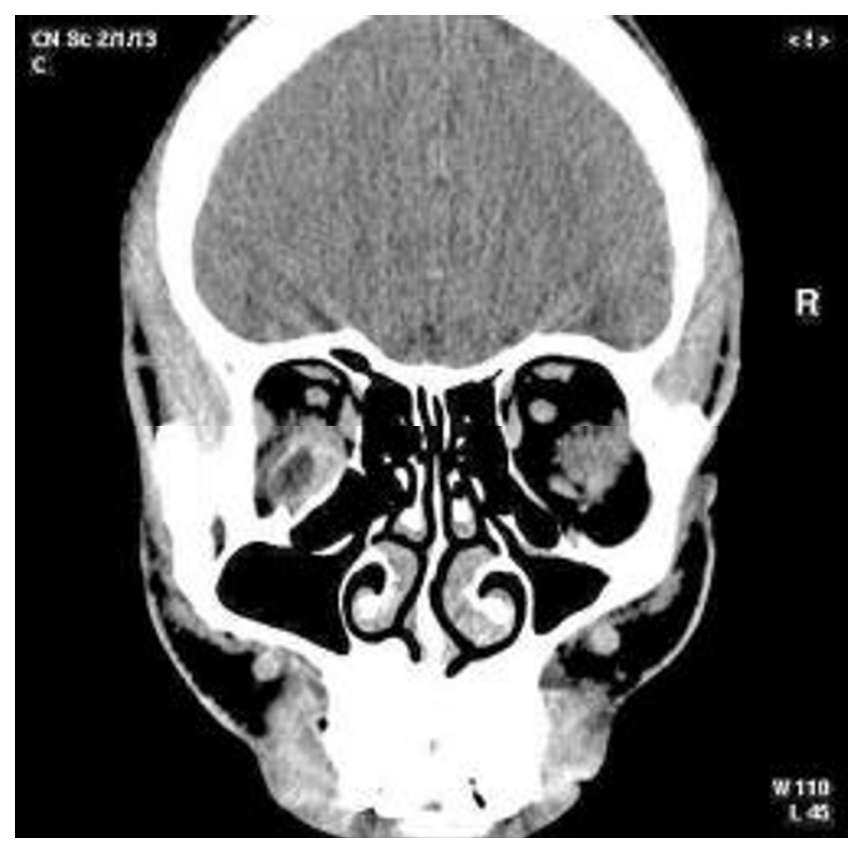

Fig. 1 : Coronal CECT orbit shows soft tissue density enhancing mass involving the belly of left inferior rectus muscle with sparing of optic nerve.

orbital reactive lymphoid hyperplasia (pseudolymphoma) and is not a lymphoid tumour.

Corticosteroids are the mainstay of therapy and are administered for several months to ensure remission. Once started, the symptoms resolve quickly. The response to steroids is so pathognomonic, that the diagnosis is often made retrospectively. Patients with multiple recurrences, or those unresponsive to therapy, should have biopsy samples taken to rule out leukaemia or lymphoma. Radiotherapy may be used in patients who fail to respond to steroids or have a rapidly progressive course. In patients who are refractory to both corticosteroids and radiotherapy, anecdotal reports have suggested the use of chemotherapeutic agents such as cyclophosphamide, methotrexate, and cyclosporine [5].

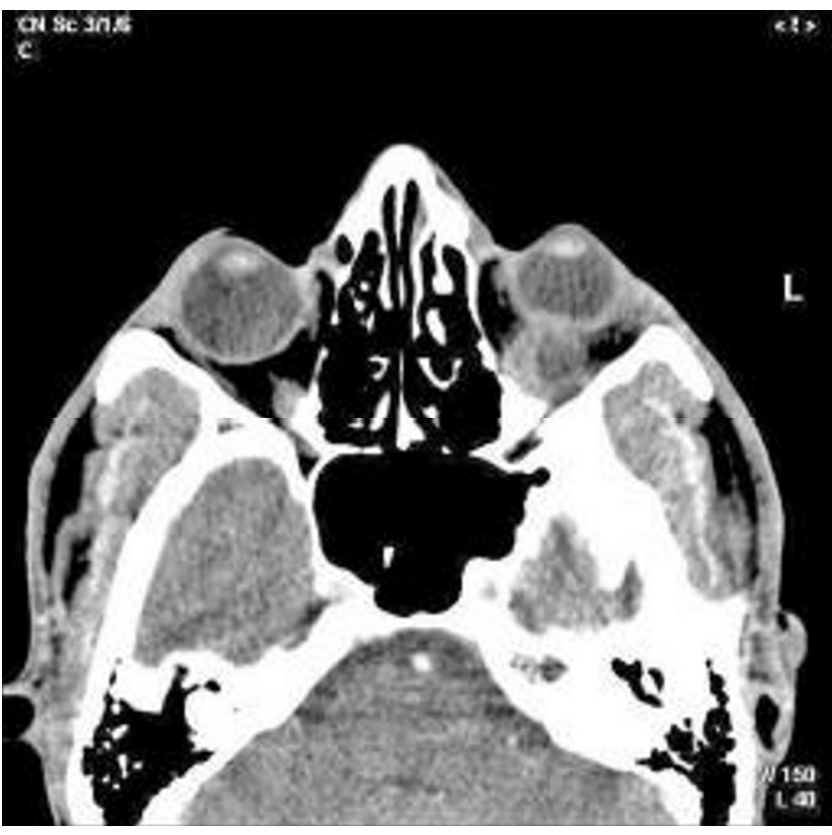

Fig. 2 : Axial CECT orbit shows the retrobulbar mass involving the tendinous insertion of inferior rectus muscle and proptosis of left globe.

Conflicts of Interest

None identified

\section{References}

1. Yuen SJ, Rubin PA. Idiopathic orbital inflammation: ocular mechanisms and clinicopathology. Ophthalmol Clin North Am 2002; 15:121-6.

2. Zurlo A, Sancesario G, Bernardi G, Loasses A. Orbital Pseudotumour: Case report and literature review. Tumori 1998; 85: $68-70$

3. Weber AL, Romo LV, Sabates NR. Pseudotumour of the orbit. Clinical, pathologic, and radiologic evaluation. Radiol Clin North Am 1999;37:151-68.

4. Unsold R, Ostertag C, Newton TH. Differential diagnosis of Graves' disease and orbital pseudotumour: CT findings. Klin Monatsbl Augenheilkd 1980; 177:31-47.

5. Jacobs D, Galetta S. Diagnosis and management of orbital pseudotumour. Curr Opin Ophthalmol 2002; 13:347-51. 